\title{
Iterative Design for Vibration Attenuation
}

\author{
T. Meurers and S.M. Veres \\ School of Electronic and Electrical Engineering, The University of Birmingham, Edgbaston B15 2TT, UK
}

Iterative design is introduced for disturbance compensation (IDDC) as a direct tuning method using closed-loop experimental data without modelling the plant. The method is based on numerical optimization of controller parameters through a sequence of experiments. The basic idea of this method is illustrated in simulation as well as in active control of a vibrating glass plate.

\section{INTRODUCTION}

Vibration can have various detrimental effects. For instance small amplitude vibration may cause errors in precision measurements or in manufacturing of semi-conductors. Large magnitude vibration can be a noise source, which the environment such as in the case of the sound of gas turbines. Hence it is important to develop control methods for vibration reduction.

Vibration control has by now a large amount of literature. There are two different control methods for vibration control. One way is to use passive mechanical vibration absorbers or dampers. ${ }^{1}$ Disadvantages of passive absorbers are that their effectiveness is either limited to a narrow frequency band or they need large bulks of insulating materials in the low frequency range (below $100 \mathrm{~Hz}$ ). The other way is to use active vibration control (AVC). This approach provides several advantages; e.g. better low frequency behaviour, reduction of size and weight and easy tunability over a broad band of disturbance frequencies. Active methods offer realistic and better alternatives to passive ones ${ }^{2}$ in an increasing number of applications.

The main problem in active vibration control is to tune the controller for the actuator. There are several methods to do this; e.g. adaptive feedforward (AFF) control, ${ }^{3}$ fuzzy control (FC), ${ }^{4}$ artificial neural networks (ANN) control, ${ }^{5} H_{\infty}$ feedback control (HF) ${ }^{6,7}$ based on internal models with uncertainty description. The classification of the approaches can be at least two-fold: (i) model-based (AFF, HF controllers) and (ii) model-free (FC, ANN controllers) . According to another classification, the control schemes can be: (a) feedforward, (b) feedback or (c) mixed, depending on whether a detection signal (sometimes called a reference signal), which is correlated with the source noise, is available and used. Feedforward controllers have been successfully applied in practice to aeroplane cabins, ${ }^{8}$ vehicles, ${ }^{9,10}$ helicopter vibration, ${ }^{11}$ active head-rests, ${ }^{12}$ etc. In many applications it is difficult to measure a well correlated signal with a disturbance which is not affected by the actuator feedback. In these cases the application of purely feedback control schemes would be preferable. Unfortunately feedback control schemes have so far been characterised by lower levels of vibration reductions and greater risk of instability. ${ }^{6,7,12,13}$

In 1964, Narendra and Streeter ${ }^{14}$ presented the idea of using experiments to generate estimated gradients of the control criterion for servo problems. This idea was recently revived by Hjalmarsson et al. ${ }^{15}$ to show that for linear time- invariant (LTI) single input/single output (SISO) systems and controllers a model free unbiased estimate of the gradient can be obtained. This method is known as iterative feedback tuning (IFT) ${ }^{16}$ for servo control.

In this paper a new design method is introduced for pure disturbance compensation using feedback control. An assumption is made that a stabilizing initial controller is known. In each iteration of the design a controller is to be computed with a better performance than in the previous one. Section 2 introduces the problem and gives a description of the iterative design method and explains its advantages compared with model based approaches. Section 3 illustrates the method in simulation and comments on possible limitations and advantages. Section 4 applies the method to vibration attenuation of a glass plate on a laboratory setup. Finally conclusions are drawn and future research directions are suggested.

\section{ITERATIVE DESIGN FOR DISTURBANCE COMPENSATION}

The aim of this approach is to control a plant which is described by Fig. 1. The vibration of the plant is represented by the output $y_{t}$. The aim is to attenuate the vibration at the output. The dynamics from the actuator signal $u_{t}$ to the output $y_{t}$ are described by an unknown transfer function $G(q)$ . The disturbance signal $d_{t}$ is a signal exciting the output directly without any further dynamics. This is an idealization which can suit many practical problems.

The system is controlled by a linear feedback controller with a transfer function $H(q, \theta)$. The adjustable parameters of the controller are described by the parameter vector $\theta$. The actuator signal is calculated by

$$
u_{t}=H(q, \theta) y_{t}
$$

Consider that there is no input signal. The response at the output $y_{t}$ to the disturbance signal $d_{t}$ in the closed-loop can be described by

$$
y_{t}=\frac{1}{1-G(q) H(q, \theta)} d_{t}
$$

where a positive feedback loop is used.

It is assumed that the disturbance signal $d_{t}$ is not measurable, it is only possible to measure and record the output 